\title{
Seroprevalencia y factores de riesgo de neosporosis bovina en el valle del Mantaro-Región Junín, Perú
}

\author{
Seroprevalence and risk factors of bovine neosporosis in the valley in \\ the Mantaro Valley, Junín, Peru
}

Fernando Arauco Villar ${ }^{1,2}$

\section{Resumen}

Se determinó la seroprevalencia de neosporosis bovina en hatos lecheros de las cuatro provincias que conforman el Valle del Mantaro, Región Junín, Perú, y los principales factores de riesgo involucrados, mediante la prueba ELISA. Se tomaron muestras de sangre de 425 animales en 37 hatos y se aplicó en forma paralela una encuesta epizootiológica. La prevalencia muestral general para neosporosis bovina fue $15.3 \%$, la prevalencia/hato de $12.8 \%$ y la prevalencia predial de $56.8 \%$, sin diferencias significativas entre provincias. Se identificaron como factores de riesgo para la presentación de neosporosis a la presencia masiva y constante de ratas (OR: 18.417), el inadecuado manejo sanitario (OR: 7.5) y el inadecuado manejo de personal (OR: 12.75). Se encontró asociación entre altas prevalencias de neosporosis con la presencia de vacas repetidoras en el hato, con los casos de abortos y nacimientos anómalos, y con el uso de agua de acequias para bebida.

Palabras clave: Valle del Mantaro; neosporosis bovina; prevalencia; factores de riesgo

\section{Abstract}

The seroprevalence of bovine neosporosis in dairy herds in four provinces of the Mantaro Valley, Peru and the main risk factors involved, through the ELISA test were determined. Blood samples were taken from 425 animals in 37 herds and an epizootic survey was applied in parallel. The general sample prevalence for bovine neosporosis was $15.3 \%$, the prevalence per herd was $12.8 \%$ and the herd prevalence was $56.8 \%$, without significant differences between provinces. The massive and constant presence

${ }^{1}$ Facultad de Zootecnia, Universidad Nacional del Centro del Perú, Huancayo, Perú

${ }^{2}$ E-Mail: faraucov@gmail.com

Recibido: 1 de marzo de 2018

Aceptado para publicación: 18 de agosto de 2018 
of rats (OR: 18,417), inadequate health management (OR: 7.5) and inadequate personnel management (OR: 12.75) were identified as risk factors for the presentation of neosporosis. An association was found between high prevalence of neosporosis with the presence of repeat breeder cows in the herd, with cases of abortions and anomalous births, and with the use of water from ditches and wells for drinking.

Key words: Mantaro Valley; bovine neosporosis; prevalence; risk factors

\section{INTRODUCCIÓN}

Neospora caninum está siendo considerado como uno de los principales agentes causantes de aborto en vacas (Anderson et al., 1994), y que puede provocar la muerte de terneros neonatos o nacimiento de animales enfermos con signos nerviosos o sin infección aparente y que se comportan como diseminadores de la enfermedad en el hato (Schares et al., 1998; Dubey, 1999).

Desde el primer reporte de aborto enzoótico por $N$. caninum en un establo de Arequipa (Andresen, 1997) se han realizado numerosas investigaciones sobre este parásito en el Perú; así, los estudios de Rivera et $a l$. (2000) indican que este parásito está presente en el $62 \%$ de las vacas que abortan, pero como el aborto no es un problema sanitario de denuncia obligatoria y por el poco o mal uso de los registros, no se conocen las cifras exactas de la incidencia nacional o regional de estos casos; además, la multicausalidad del problema no contribuye a realizar un diagnóstico preciso (Fredes, 2000).

Otros reportes registran valores de prevalencia que van desde $1.5 \%$ en Pucallpa hasta $57 \%$ en Arequipa, considerando a Cajamarca con $42.9 \%$, Lima $29.6 \%$, Puno $18.1 \%$ y Junín $12.8 \%$, observándose una mayor seropositividad en las principales cuencas lecheras del país (Rivera, 2001). El Servicio Nacional de Sanidad Agraria (SENASA, (2010) reporta a nivel nacional una prevalencia muestral de $20.3 \%$ y predial de $67 \%$, y para Junín de 15.8 y $70.6 \%$, respectivamen- te. En Moquegua se obtuvo una seroprevalencia de 51\% (Mamani, 2007), en Locumba, Tacna de 44\% (Alarico, 2012), mientras que en Melgar, Puno se reportó una seroprevalencia de $18 \%$ en ganado lechero al pastoreo. Así mismo, en vacas Brown Swiss de la SAIS Pachacútec, Junín, se reportó la presencia de $13 \%$ de anticuerpos contra N. caninum (Puray, 2006), y 46.7\% en vacas lecheras de la margen izquierda del Valle del Mantaro, Junín (Granados et al., 2014).

Existe escasa información sobre los factores de riesgo que pueden estar asociados a la presencia y diseminación de la neosporosis bovina; sin embargo, su conocimiento es importante para el desarrollo y aplicación de medidas para controlar la enfermedad. En Mato Grosso, Brasil, se encontró asociación entre la seropositividad de las vacas y las fuentes naturales de agua potable, aunque no hubo asociación con la presencia de perros (Justo et al., 2013). El uso de estanques de agua puede ser un factor de riesgo para la infección del ganado con N. caninum (Ould et al., 1999), pues se tiene información que los ooquistes de $N$. caninum pueden contaminar fuentes de agua superficial (Dubey et al., 2003). Como la transmisión vertical del $N$. caninum es muy eficiente, la cría de vaquillas de reemplazo propias, en lugar de comprarlas de otros hatos, apoya la idea de que la prevalencia existente en una manada puede perdurar durante muchos años (Frössling et al., 2005; Stenlund et al., 2003). El riesgo de infección fue también mayor en hatos donde la entrada de animales fue frecuente (hatos abiertos) al compararlos con los hatos cerrados (Fort, 2011). 
Este estudio presenta parte de los resultados de un estudio marco que evaluó la presencia de dos enfermedades abortígenas en el Valle del Mantaro, Diarrea Viral Bovina y Neosporosis. Este trabajo pretende conocer la seroprevalencia de $N$. caninum en los hatos bovinos lecheros en las provincias de Huancayo, Chupaca, Concepción y Jauja, que conforman el Valle del Mantaro, y los factores de riesgo involucrados en su presentación.

\section{Materiales y Métodos}

El estudio se realizó en establos bovinos lecheros ubicados en las cuatro provincias que conforman el Valle del Mantaro Región Junín, Perú (Huancayo, Chupaca, Concepción y Jauja), tanto en sus márgenes izquierda y derecha, y sus zonas de influencia en los pisos altitudinales medio y zona alta. El Valle del Mantaro se encuentra a una altitud promedio de $3330 \mathrm{msnm}$. Los predios o hatos lecheros fueron seleccionados independientemente del sistema de manejo empleado, que aceptaran los términos establecidos en el documento de consentimiento informado. Para la selección de los animales, se consideró que las vacas estuviesen en ordeño, indistintamente de la raza y edad.

El promedio de la población bovina en los hatos en estudio fue 34.5 (6 hatos con rango 1-10 animales, 16 con 11-30, 5 con 3160 , y 10 con $>60$ ). El número de vacas muestreadas por hato fue de 16.1 (6-30) en Huancayo, 4.1 (2-12) en Chupaca, 11.7 (120) en Concepción y 13.6 (4-36) en Jauja. El número promedio de partos por vaca fue de $2.8(1-13)$.

El tamaño muestral se determinó empleando la fórmula $n=\left(z_{\mathrm{a} / 2}\right)^{2}\left(\mathrm{pq} / \mathrm{L}^{2}\right)$, donde $n$ : tamaño muestral mínimo, Z: nivel de confianza (95\%), $p$ : proporción referencial, $q$ : 1- $p, \mathrm{y}$ $L$ : error máximo permisible (5\%) (Daniel, 1996), considerando una proporción referencial de $25 \%$. Se tomaron 425 muestras, distribuidas proporcionalmente al tama- ño poblacional de vacas en las cuatro provincias (CENAGRO-Junín, 2012), correspondiendo $145,33,125$ y 122 muestras a Huancayo, Chupaca, Concepción y Jauja, respectivamente. Las unidades primarias de muestreo fueron los hatos o predios seleccionados aleatoriamente, tomando en cuenta los datos de la población bovina de las provincias (Huancayo; 9, Concepción: 11; Jauja: 9; Chupaca: 8), haciendo un total de 37 hatos.

Las muestras de sangre se obtuvieron por punción directa de la arteria coccígea media, utilizando tubos Vacutainers estériles de $10 \mathrm{ml}$ sin anticoagulante. El suero obtenido se conservó en viales de $2 \mathrm{ml}$ en congelación a $-20{ }^{\circ} \mathrm{C}$ hasta su procesamiento.

La detección y cuantificación de anticuerpos específicos frente a los taquizoítos de $N$. caninum se hizo mediante ELISA indirecto utilizando el kit CIVTEST Bovis Neospora (Lab. Hipra) complementado con el kit cELISA VMRD 280-5 N. caninum antibody Test Kit (Lab. Multivet), siguiendo los protocolos recomendados por los laboratorios fabricantes. Los controles positivo y negativo se procesaron por duplicado. Las lecturas de las absorbancias se hicieron en la lectora de placas Biotek Modelo ELx800.

Se registró la población bovina por hato, raza, edad y número de parto de los animales, número de perros por predio, el sistema de producción (intensivo, otros), tipo de hato (cerrado, donde no se adquieren animales de otros establecimientos y se desprende de sus animales ocasionalmente; abierto, donde incorpora animales de cualquier procedencia y vende o moviliza animales bajo diferentes modalidades), tipo de crianza (solo bovinos, crianza mixta), cercanía a centros poblados (sí, no), origen de los reemplazos (propios, externos), tipo de instalaciones (adecuadas, inadecuadas), presencia de animales silvestres, excepto ratas (sí, no), presencia de ratas (de manera ocasional o esporádica, o de manera permanente y masiva), tipo de servicio reproductivo (monta natural, inseminación artificial, mixto), tipo de semen (nacional, im- 
portado), manejo de placentas y fluidos uterino posparto (adecuado, inadecuado), higiene (adecuada, inadecuada), manejo sanitario (adecuado, inadecuado), manejo de personal (adecuado, inadecuado). Así mismo, se registró la presencia de vacas repetidoras en los hatos durante en el último año $(0,1-3,>3$ casos), así como problemas de abortos y de nacimientos de terneros débiles o con malformaciones congénitas reportados en los últimos tres años. El promedio de perros/hato fue 3.1 (rango 0-9) con una moda de 2.

La prevalencia (\%) de neosporosis se calculó mediante la fórmula $\left(\mathrm{N}^{\mathrm{o}}\right.$ muestras positivas / total de muestras) * 100. Para el cálculo de la prevalencia predial se consideró el número de hatos con al menos un animal seropositivo a neosporosis sobre el total de hatos evaluados. Para el análisis de los datos se utilizó el paquete estadístico SPSS v. 21. Se determinó la prevalencia con sus respectivos intervalos de confianza, se hizo la correlación de Spearman y el análisis de regresión logística binaria, así como tablas de contingencia con Chi cuadrado y medidas de asociación (riesgo) como Odds Ratio (OR). Además, se aplicó una encuesta epizootiológica que fue validada en su contenido por un panel de expertos, empleando el Método Delphi y la prueba no paramétrica de Kendall (Pérez, 1993).

Se proporcionó a los propietarios o responsables de los predios de información pertinente sobre el trabajo a desarrollarse y el destino de las muestras colectadas, con el compromiso de confidencialidad y reserva del caso, firmándose la correspondiente Ficha de Consentimiento Informado.

\section{Resultados}

\section{Prevalencia de Neosporosis}

La prevalencia general fue de $15.3 \%$ (65/425), la prevalencia por hato de $12.8 \%$ y la prevalencia predial de 56.8\% (16/37)(Cua- dro 1), sin que hubiera diferencias significativas entre provincias para los diferentes tipos de prevalencia.

Los resultados de prevalencia fueron categorizados por cuartiles dentro del rango de $0-52 \%$ (Arauco, 2015), encontrándose que el $59.56 \%$ de los hatos presentó niveles de prevalencia bajos y el $86.5 \%$ fue bajo-moderado bajo pues presentaron niveles de prevalencia de neosporosis entre $0 \mathrm{y}<27 \%$ (Cuadro 2).

\section{Factores Ambientales y de Manejo}

El tamaño de la población bovina por hato, raza, edad y numero de parto de los animales muestreados y el número de perros por hato no presentaron correlaciones significativas con la prevalencia de neosporosis por hato.

El nivel de riesgo de variables relacionadas al manejo de los animales y factores ambientales con la presentación de casos de prevalencias altas de neosporosis en el hato se muestran en el Cuadro 3. La higiene inadecuada, e inadecuados manejo sanitario y del personal, así como la presencia de ratas en el predio son factores de riesgo para la presentación de neosporosis.

El sistema de producción (intensivo vs otros), tipo de hato (cerrado vs. abierto), origen de los reemplazos (propios vs. externos), tipo de instalaciones (adecuadas vs. inadecuadas), presencia de animales silvestres, excepto ratas (sí vs. no), tipo de servicio reproductivo (monta natural vs. inseminación artificial vs. mixto), tipo de semen (nacional vs. importado), y manejo de placentas y fluidos uterino posparto (adecuado vs. inadecuado) no constituyeron factores de riesgo para la presentación de neosporosis.

Al asociar las causas de saca con el porcentaje de prevalencia de neosporosis/hato se encontró que hatos con mayores niveles de neosporosis reportan como principal causa de saca la baja producción de las vacas, 
Cuadro 1. Prevalencia de neosporosis muestral, predial y por hato por provincia

\begin{tabular}{lccccc}
\hline Provincia & $\begin{array}{c}\text { Muestras } \\
(\mathrm{n})\end{array}$ & Hatos (n) & $\begin{array}{c}\text { Prevalencia } \\
\text { muestral }(\%)\end{array}$ & $\begin{array}{c}\text { Prevalencia } \\
\text { predial }(\%)\end{array}$ & $\begin{array}{c}\text { Prevalencia } \\
\text { por hato (\%) }\end{array}$ \\
\hline Huancayo & 145 & 9 & 18.6 & 55.6 & 15.2 \\
Chupaca & 33 & 8 & 15.2 & 25.0 & 10.4 \\
Concepción & 125 & 11 & 16.0 & 81.8 & 16.8 \\
Jauja & 122 & 9 & 10.7 & 45.5 & 7.4 \\
\hline Total & 425 & 37 & $15.1 \pm 3.3$ & $56.8 \pm 23.6$ & $12.8 \pm 4.3$ \\
\hline
\end{tabular}

Cuadro 2. Niveles de prevalencia de neosporosis en hatos bovinos del Valle del Mantaro, Junín, Perú

\begin{tabular}{lcc}
\hline \multirow{2}{*}{ Nivel } & \multicolumn{2}{c}{ Hatos } \\
\cline { 2 - 3 } & $\mathrm{n}$ & $\%$ \\
\hline Bajo & 22 & 59.5 \\
Moderado Bajo & 10 & 27.0 \\
Moderado Alto & 2 & 5.4 \\
Alto & 3 & 8.1 \\
\hline Total & 37 & 100.0 \\
\hline
\end{tabular}

los hatos con prevalencias moderadas reportaron los problemas reproductivos y otra causal más, mientras que hatos con prevalencias bajas reportaron la edad de los animales como principal causa de saca.

\section{Problemas Reproductivos}

El $40.5 \%$ de los hatos reportaron abortos, $5.4 \%$ nacimientos anómales, $32.5 \%$ ambos tipos de ocurrencias y $21.6 \%$ no reportaron este tipo de problemas. No se encontró asociación significativa entre las prevalencias altas de neosporosis con los casos de abortos y nacimientos anómalos, y solo hubo asociación significativa entre los nacimientos de terneros con malformaciones congénitas y prevalencias moderadas y bajas de neosporosis. Así mismo, hatos que reportaron más de tres casos de retención de placenta fueron aquellos con prevalencias altas de neosporosis, aunque esta asociación no fue significativa.

Cuadro 3. Factores de riesgo para la presentación de neosporosis de ganado bovino en el Valle del Mantaro

\begin{tabular}{lcc}
\hline Variable & Odds ratio (OR) & IC 95\% \\
\hline Presencia de ratas (ocasional/esporádica - & 18.417 & $3.495-97.055$ \\
permanente/masiva) & 17.5 & $3.016-101.54$ \\
Higiene (adecuada - inadecuada) & 12.75 & $2.651-61.322$ \\
Manejo de personal (adecuado - inadecuado) & 7.5 & $1.715-32.796$ \\
Manejo sanitario (adecuado - inadecuado) & 0.210 & $0.046-0.959$ \\
Tipo de crianza (solo bovinos - crianza mixta) & 0.142 & $0.033-0.610$ \\
Cercanía a centros poblados (sí - no) & & \\
\hline
\end{tabular}




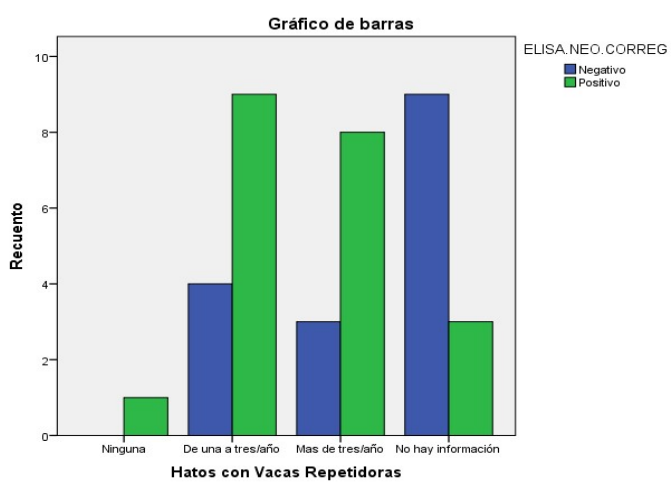

Figura 1. Frecuencias de vacas repetidoras por hato y casos de neosporosis

Considerando la frecuencia de presentación de casos de vacas repetidoras en los hatos evaluados en el último año, 13 de ellos (52\%) registraron de $1-3$ casos, 11 (44\%) registraron más de 3 casos y un hato (4\%) no los tuvo. El mayor número de hatos con casos positivos de neosporosis estuvo asociado con la presencia de 1-3 y $>3$ casos de vacas repetidoras (Figura 1).

\section{Discusión}

La neosporosis bovina en el Valle del Mantaro es una enfermedad que está presente en la ganadería lechera, aunque en menor proporción que en otras partes del país. En las cuatro provincias del Valle del Mantaro hay predominio de prevalencias bajas de neosporosis bovina, no habiendo diferencia significativa entre provincias. No obstante, la subvaloración de la enfermedad entre los productores del valle puede dar lugar a que se descuide su control. Por otro lado, puede haber una superposición de noxas, especialmente si coinciden DVB y neosporosis, y en estos casos la tasa de abortos suele ser más alta que los casos de abortos generados por solo N. caninum (Meléndez et al., 2010).

La prevalencia muestral (15.3\%) fue similar a las prevalencias reportadas para Junín de $15.8 \%$ y para la SAIS Pachacútec,
Junín de 12.8\% (Puray et al., 2006), mientras que en la provincia de Melgar, Puno, se reportó 18.1\% (Atocsa et al., 2005). Por otro lado, Granados et al. (2014) reportó para la provincia de Concepción una prevalencia de $46.7 \%$, mientras que en el presente trabajo fue de $16.0 \%$.

La baja y no significativa correlación entre número de perros en el predio con la tasa de prevalencia de neosporosis se encuentra acorde con otros resultados (Romero y Frankena, 2003; Ogawa et al., 2005; Bañales et al., 2006; Escalona et al., 2010), a pesar de la identificación del perro doméstico (Canis familiaris) como hospedero definitivo de $N$. caninum. Esto sugiere que la principal forma de transmisión de la neosporosis en los hatos evaluados es de tipo vertical, para la cual sólo es necesario que las hembras reproductoras de los hatos estén infectadas, sin necesidad de que sean sometidas a reinfecciones (Paré et al., 1996; Wouda et al., 1998).

Se debe tener presente que el ciclo biológico del parásito involucra variados hospederos intermediarios, entre los que se incluyen, aparte de los bovinos a ovinos, caprinos y equinos (Dubey, 2003); por tanto, la exposición de más potenciales hospedadores intermediarios al riesgo de infección puede ser compatible con mayores prevalencias de la enfermedad.

La higiene inadecuada (limpieza y desinfección de ambientes e implementos, eliminación de excretas, uso de estercoleros, desratizaciones, desinsectaciones, etc.) fue un factor de riesgo (OR: 17.5) en la presentación de neosporosis. El 10.8\% de hatos realizaban buenas prácticas de higiene en sus instalaciones (limpieza y desinfección de ambientes e implementos, eliminación de excretas, uso de estercoleros, desratizaciones, desinsectaciones, etc.). El mayor riesgo es por la mayor oportunidad de contacto de los animales susceptibles con los ooquistes del parásito, producto de la contaminación con heces de perros infectados, siendo importan- 
te en este caso la transmisión horizontal. Así mismo, el deficiente manejo sanitario (no hay plan de dosificación ni vacunación establecido en el hato, no se realizan pruebas diagnósticas, no se separa a los animales enfermos, no hay equipo ni botiquín veterinario y no se solicita asesoramiento veterinario frente a los problemas sanitarios) fue determinante para la presentación de mayor número de casos positivos de neosporosis (OR: 7.5).

El manejo inadecuado del personal a cargo de los animales constituyó un factor de riesgo (OR: 12.75) para la presentación de casos positivos de neosporosis. Las buenas prácticas en el manejo del personal incluyen que el mismo debe gozar de buena salud (con certificación médica anual), capacitaciones continuas para la mejora de su labor y para preservar la salud humana, de los animales a su cargo y como obtener un producto inocuo. La encuesta reveló que el personal es mayormente de tipo eventual, sin muchos beneficios laborales e incentivos para una labor eficiente, lo que se traduce por lo general en un desinterés y desmotivación en sus labores (Uribe et al., 2011).

En la encuesta realizada se reportó la presencia de ratas en las instalaciones de todos los predios (de manera ocasional o esporádica, o de manera permanente y masiva), constituyendo la presencia de estos roedores de manera constante y masiva un factor de riesgo (OR: 18.417) para neosporosis. Se ha reportado presencia de ADN de $N$. caninum en ratas y ratones infectados naturalmente, lo que sugiere que estos animales pueden ser importantes fuentes de infección para los carnívoros hospedadores de $N$. caninum (Dubey et al., 2007), aunque el mayor problema con estos roedores es su rol como vectores mecánicos de los ooquistes del parásito, lo cual favorecería la transmisión horizontal de la enfermedad (Ortega-Mora et al., 2006).

El estudio indica que el ganado con valores altos de prevalencia de neosporosis suele usar las acequias circundantes a los potreros de pastoreo, aunque el nivel de asociación no fue significativo. Esta fuente de agua aumenta el riesgo de contaminación con ooquistes de $N$. caninum. En un estudio se identificó ADN de $N$. caninum en el $87-93 \%$ de muestras de agua provenientes de pozos y bebederos (Sierra et al., 2011). El uso de estanques de agua en lugar de usar el suministro de la red pública de agua potable puede ser un factor de riesgo para la infección del ganado con $N$. caninum, (Ould et al., 1999).

No se pudo establecer que la presencia de vacas repetidoras en el hato constituya un factor de riesgo para la presentación de neosporosis; sin embargo, según los resultados obtenidos, el mayor número de hatos con casos positivos de neosporosis estuvo asociado con la presencia de 1-3 y $>3$ casos de vacas repetidoras. Oviedo et al. (2007) reportaron en Córdova, Colombia, que el 9.8\% de los animales seropositivos a $N$. caninum fueron vacas repetidoras de celo.

\section{Conclusiones}

- La prevalencia muestral general para neosporosis bovina fue $15.3 \%$, la prevalencia/hato de $12.8 \%$ y la prevalencia predial de $56.8 \%$.

- Los factores de riesgo identificados para la presentación de neosporosis fueron la presencia masiva y constante de ratas (OR: 18.417), el inadecuado manejo sanitario (OR: 7.5) y de manejo de personal (OR: 12.75).

- La asociación entre altas prevalencias de neosporosis con la presencia de vacas repetidoras, con casos de abortos y nacimientos anómalos, y con el uso de agua de acequias para bebida no fue significativa.

\section{Literatura Citada}

1. Alarico ZDA. 2012. Determinación de seroprevalencia de anticuerpos a Neospora caninum en bovinos de le- 
che del distrito de Locumba-Tacna. Tesis de Médico Veterinario Zootecnista. Tacna: Univ. Nacional Jorge Basadre Grohmann. $134 \mathrm{p}$.

2. Anderson ML, Barr BC, Conrad PA. 1994. Protozoal causes of reproductive failure in domestic ruminants. Vet Clin N Am-Food A 10: 439-461. doi: 10.1016/ S0749-0720(15)30531-4

3. Andresen H. 1997. Neosporosis: enfermedad emergente en los animales domésticos. MV Rev Cs Vet 13: 19-21.

4. Arauco VF. 2015. Epidemiología y caracterización de los factores de riesgo de diarrea viral bovina y neosporosis en bovinos del Valle del Mantaro - región Junín. Tesis Doctoral. Lima, Perú: Univ. Nacional Mayor de San Marcos. 179 p.

5. Atoccsa J, Chávez A, Casas E, Falcón N. 2005. Seroprevalencia de Neospora caninum en bovinos lecheros al pastoreo en la provincia de Melgar, Puno. Rev Inv Vet Perú 16: 71-75. doi: 10.15381/rivep.v16i1.1541

6. Bañales P, Fernandez L, Repiso MV, Gil A, Dargatz DA, Osawa T. 2006. A nationwide survey on seroprevalence of Neospora caninum infection in beef cattle in Uruguay. Vet Parasitol 139: 1520. doi: 10.1016/j.vetpar.2006.03.004

7. [CENAGRO-JUNIN] Censo Nacional Agropecuario, Junín. 2012. Resultados definitivos del IV Censo Nacional Agropecuario. [Internet]. Disponible en: http:/ /censos.inei.gob.pe/cenagro/tabulados/

8. Daniel WW. 1996. Bioestadística: base para el análisis de las ciencias de la salud. $5^{\circ}$ ed. México.. Limusa. 878 p.

9. Dubey JP. 1999. Neosporosis in cattle: biology and economic impact. J Am Vet Med Assoc 214: 1160-1163.

10. Dubey JP. 2003. Review of Neospora caninum and neosporosis. Kor J Parasiol 41: 1-16. doi: 10.3347/kjp.2003.41.1.1

11. Dubey JP1, Zarnke R, Thomas NJ, Wong SK, Van Bonn W, Briggs $M$, Davis JW, et al. 2003. Toxoplasma gondii, Neospora caninum, Sarcocystis neurona, and Sarcocystis canislike infections in marine mammals. Vet
Parasitol 116: 275-296. doi: 10.1016/ S0304-4017(03)00263-2

12. Dubey JP, Schares G, Ortega-Mora LM. 2007. Epidemiology and control of neosporosis and Neospora caninum. Clin Microbiol Rev 20: 323-367. doi: 10.1128/CMR.00031-06

13. Escalona J, García F, Mosquera O, Vargas F, Corro A. 2010. Factores de riesgo asociados a la prevalencia de neosporosis bovina en el municipio Bolívar del estado Yaracuy, Venezuela. Zootecnia Trop 28: 201-211.

14. Fort M. 2011. Neospora caninum: Estudio seroepidemiológico en bovinos de la provincia de La Pampa. Pub. Téc. INTA 52. [Internet]. Disponible en: https:/ /inta.gob.ar/documentos/neosporacaninum-estudio-seroepidemiologico-enbovinos-de-la-provincia-de-la-pampa

15. Fredes MF. 2000. La neosporosis una parasitosis emergente. TecnoVet 6(3). [Internet]. Disponible en: https:// revistas.uchile.cl/index.php/RT/issue/ view/569

16. Frössling J, Uggla A, Björkman C. 2005. Prevalence and transmission of Neospora caninum within infected Swedish dairy herds. Vet Parasitol 128: 209-218. doi: 10.1016/j.vetpar.2004.12.006

17. Granados S, Rivera H, Casas E, Suárez F, Carana C, Chávez A. 2014. Frecuencia de Neospora caninum en bovinos lecheros de cuatro distritos del Valle del Mantaro, Junín. Rev Inv Vet Perú 25: 58-64. doi: 10.15381/ rivep.v25i1.8468

18. Justo RV, Manfio JB, Galhardo JA, Garcia JL, Campos AK. 2013. Seroepidemiological inquiry on bovine neosporosis in northern Mato Grosso state, Brazil. Semin-Cienc Agrar 34(Suppl 2): 3897-3902. doi: 10.5433/ 1679-0359.2013v34n6Sup12p3897

19. Mamani J. 2007. Seroprevalencia de Neospora caninum en bovinos lecheros en Moquegua, distrito de Moquegua, provincia Mariscal Nieto y departamento de Moquegua-2007. Tesis de Médico 
Veterinario Zootecnista. Arequipa, Perú: Univ. Católica Santa María. 85 p.

20. Meléndez R, Valdivia A, Rangel E, Díaz E, Segura J, Guerrero A. 2010. Factores de riesgo asociados a la presencia de aborto y desempeño reproductivo en ganado lechero de Aguascalientes, México. Rev Mec Cienc Pecuarias 1: 391-401.

21. Ogawa L, Freire RL, Vidotto $O$, Gondim LFP, Navarro IT. 2005. Occurrence of antibodies to Neospora caninum and Toxoplasma gondii in dairy cattle from the northern region of the Paraná State, Brazil. Arq Bras Med Vet Zoo 57: 312-316. doi: 10.1590/S010209352005-000300006

22. Ortega-Mora L, Fernández-García A, Gómez M. 2006. Diagnosis of bovine neosporosis: recent advances and perspectives. Acta Parasitol 51: 1-14. doi: 10.2478/s11686-006-0001-0

23. Ould-Amrouche A, Klein F, Osdoit C, Mohammed HO, Touratier A, Sanaa M, Mialot JP. 1999. Estimation of Neospora caninum seroprevalence in dairy cattle from Normandy, France. Vet Res 30: 531-538.

24. Oviedo ST, Betancur HC, Mestra PA, Gonzáles TM, Reza GL, Calonge GK. 2007. Estudio serológico sobre neosporosis en bovinos con problemas reproductivos en Montería, Córdoba, Colombia. Rev MVZ Córdoba 12: 929-933. doi: $10.21897 / \mathrm{rmvz} .437$

25. Paré J, Thurmond MC, Hietala SK. 1996. Congenital Neospora caninum infection in dairy cattle and associated calfhood mortality. Can J Vet Res 60: 133-139.

26. Pérez SP. 1993. Validación de cuestionario para la medición de la satisfacción de los clientes de la DCI. Gestión de Calidad DCI del CNCI, Cienfuegos. Cuba. [Internet]. Disponible en: http:// www.monografias.com/trabajos93/ validacion-cuestionario-medicion- satisfaccion-clientes-dci/validacioncuestionario-medicion-satisfaccion-clientes-dci.shtml

27. Puray CN, Chávez A, Casas E, Falcón N, Casas G 2006. Prevalencia de Neospora caninum en bovinos en empresa ganadera de la sierra central del Perú. Rev Inv Vet Perú 17: 189-194. doi: 10.15381/rivep.v17i2.1542

28. Rivera GH. 2001. Causas frecuentes de aborto bovino. Rev Inv Vet Perú 12: 117-122.

29. Rivera GH, Nelson D, Tabacchi L. 2000. Neospora caninum y otros agentes en fetos abortados de bovinos lecheros del valle de Lima. Rev Inv Vet Perú 11: 1-7. doi: 10.15381/rivep.v11i1.6766

30. Rojas CM. 2009. Neosporosis: parasitosis emergente en la ganadería peruana. [Internet]. Disponible en: http:/ /mrojas.perulactea.com/2009/02/23/ neosporosis-parasitosis-emergente-enla-ganaderia-peruana/

31. Romero JJ, Frankena K. 2003. The effect of the dam-calf relationship on serostatus to Neospora caninum on 20 Costa Rican dairy farms. Vet Parasitol 114: 159-171. doi: 10.1016/S03044017(03)00135-3

32. Schares G, Peters M, Wurm R, Bärwald A, Conraths FJ. 1998. The efficiency of vertical transmission Neospora caninum in dairy cattle analysed by serological techniques. Vet Parasitol 80: 87-98. doi: 10.1016/S03044017(98)00195-2

33. [SENASA] Servicio de Sanidad Agraria. 2010. Caracterización de la diarrea viral bovina, neosporosis bovina y rinotraqueitis infecciosa bovina en el Perú. [Internet]. Disponible en: https:// www.senasa.gob.pe/senas a/ descargasarchivos/jer/BOVINOS/Caracterizacion $\%-20 \mathrm{DVB} \% 20 \mathrm{NB} \%$ 20y\%20RIB.pdf

34. Sierra RC, Medina-Esparza L, Ramos M, García-Vázquez, Z, Cruz-Vázquez C. 2011. Factores de riesgo asociados 
a la seroprevalencia de anticuerpos a Neospora caninum en ganado lechero de Aguascalientes, México. Rev Mex Cienc Pecu 2: 15-24.

35. Stenlund S, Kindahl H, Uggla A, Björkman C. 2003. A long-term study of Neospora caninum infection in a Swedish dairy herd. Acta Vet Scand 44: 63-71. doi: 10.1186/1751-0147-44-63
36. Uribe F, Zuluaga AF, Valencia L, Murgueitio E, Ochoa L. 2011. Buenas prácticas ganaderas. Manual 3. Colombia: CIPAV. $82 \mathrm{p}$.

37. Wouda W, Moen AR, Schukken YH. 1998. Abortion risk in progeny of cows after a Neospora caninum epidemic. Theriogenology 49: 1311-1316. doi: 10.1016/S0093-691X(98)00078-8 